\title{
Atomic-scale Structure and Morphology of $\gamma$-FeOOH Particles Formed during Corrosion of Fe-based Alloys in Aqueous Solution*
}

\author{
Katsuya Inoue ${ }^{\dagger}$ \\ Institute of Multidisciplinary Research for Advanced Materials, \\ Tohoku University, 2-1-1 Katahira, Aoba-ku, Sendai 980-8577, Japan \\ Research Fellow of the Japan Society for the Promotion of Science \\ Kozo Shinoda \\ Institute of Multidisciplinary Research for Advanced Materials, \\ Tohoku University, 2-1-1 Katahira, Aoba-ku, Sendai 980-8577, Japan, \\ Masatoshi Saito \\ School of Health Sciences, Faculty of Medicine, Niigata University, \\ 1, Asahimachi-dori, Niigata, Niigata, 951-8518, Japan \\ Shigeru Suzuki and Yoshio Waseda \\ Institute of Multidisciplinary Research for Advanced Materials, \\ Tohoku University, 2-1-1 Katahira, Aoba-ku, Sendai 980-8577, Japan
}

(Received 5 November 2007; Accepted 25 January 2008; Published 13 February 2008)

\begin{abstract}
Quantitative X-ray structural analysis is used for characterizing the atomic-scale structure of $\gamma$-FeOOH particles; these particles are formed by immersing pure iron, iron-aluminum, iron-silicon, and iron-phosphorus alloys into an aqueous solution of sodium chloride. The morphology of these $\gamma$-FeOOH particles is observed by transmission electron microscopy, and their bonding structure is analyzed using Fourier transform infrared spectroscopy. The realistic atomic-scale structure of the $\gamma-\mathrm{FeOOH}$ particles is estimated by fitting the interference functions with the help of the reverse Monte Carlo simulation technique. The results show that the linkages of fundamental $\mathrm{FeO}_{6}$ octahedral units in $\gamma$-FeOOH had underwent deviation from the ideal crystal structure. The structural deviation and morphological change of the particles were considered to be due to the incorporation of foreign ions during the formation of these particles in the aqueous solution. [DOI: 10.1380/ejssnt.2008.49]
\end{abstract}

Keywords: Iron corrosion products; Atomic-scale structure; X-ray diffraction; Transmission electron microscopy; Infrared spectroscopy

\section{INTRODUCTION}

As the anodic and cathodic reactions occur on the steel surface under a humid atmosphere [1, 2], corrosion products are precipitated from ferric and ferrous ions and hydroxyl ions formed from steel by these reactions [3]. The species and morphology of corrosion products are influenced by the reaction conditions such as temperature, humidity, and the presence of chloride ions and sulfate ions in the atmosphere. Foreign cations and anions originating from alloying elements are also likely to affect the formation of corrosion products, and the resulting species and fraction of components in corrosion products are influenced by ferrous and ferric ions, foreign ions, and so on $[1,2]$. As a layer of corrosion products formed on the surface of weathering steel is known to reduce the corrosion rate of the steel substrate, the influence of alloying elements on the structure of corrosion products must be characterized. The weathering steel is low-alloy steel containing elements such as chromium, copper, and phosphorus that may play an important role in the formation of a protective rust layer $[4,5]$.

The main components of corrosion products formed

*This paper was presented at 6th International Symposium on Atomic Level Characterizations for New Materials and Devices, Kanazawa, Japan, 28 October-2 November, 2007.

$\dagger$ Corresponding author: inoue@mail.tagen.tohoku.ac.jp during atmospheric corrosion are the ferric oxyhydroxides, $\alpha$-FeOOH and $\gamma$-FeOOH, whose structure is described by $\mathrm{FeO}_{6}$ octahedral units. They are linked to each other by corners, edges, or faces to form different structural arrays that are maintained even in the amorphous state [6]. As the linkages of the octahedral units are strongly related with the morphology and stability of ferric oxyhydroxides, the atomic-scale structure of ferric oxyhydroxides containing foreign ions has been studied [7-12]. In these studies, the extended X-ray absorption fine structure (EXAFS) $[13,14]$ and anomalous X-ray scattering (AXS) [15] are measured for characterizing the atomic-scale structure of different ferric oxyhydroxides. Although the EXAFS method is used for characterizing the local structure of corrosion products, the structural information obtained by EXAFS seems to be in a very short range; therefore, the linkage of the $\mathrm{FeO}_{6}$ octahedral units cannot be discussed on this basis. In contrast, the AXS method coupled with reverse Monte Carlo (RMC) simulation is useful for characterizing the atomic-scale arrangement of ferric oxyhydroxides [16-18]. The results obtained by these structural analyses show that the linkages of $\mathrm{FeO}_{6}$ octahedral units in ferric oxyhydroxides are influenced by foreign cations and anions.

As aluminum, silicon, and phosphorus - whose atomic numbers are close - are important alloying elements in steel, it is important to study the influence of these elements on the structure and morphology of the corrosion products. The objective of this study is to investigate the influence of aluminum, silicon, and phosphorus on 
the structure of $\gamma$-FeOOH, which was formed during the corrosion of pure iron, iron-aluminum, iron-silicon, and iron-phosphorus alloys in aqueous solution. Quantitative $\mathrm{X}$-ray structural analysis coupled with RMC simulation and Fourier transform infrared spectroscopy (FT-IR) are used to analyze the influence of these elements on the atomic-scale structure of $\gamma$-FeOOH. The morphology of the corrosion products was observed by transmission electron microscopy (TEM).

\section{EXPERIMENTAL}

\section{A. Sample preparation}

Approximately 0.3-mm-thick sheets of pure iron $(\mathrm{Fe})$, iron - 3 mass \% aluminum ( $\mathrm{Fe}-\mathrm{Al}$ ) alloy, iron - 2 mass\% silicon (Fe-Si) alloy, and iron - 0.58 mass\% phosphorus (Fe-P) alloy were used as starting materials. The sheets were cut such that they had dimensions of $30 \mathrm{~mm} \times 25 \mathrm{~mm}$, and they were immersed into aqueous solutions of sodium chloride ( $\mathrm{NaCl}, 2$ mass\%). Corrosion products consisting mainly of $\gamma$-FeOOH particles were formed in open 150$\mathrm{ml}$ glass bottles for 14 days at room temperature. The $\gamma$-FeOOH particles were filtered and dried at room temperature. The corrosion products, prepared from Fe, Fe$\mathrm{Al}$ alloy, Fe-Si alloy, and Fe-P alloy in the solution, are referred to as $\gamma$-Fe-Rust, $\gamma$-FeAl-Rust, $\gamma$-FeSi-Rust, and $\gamma$-FeP-Rust, respectively. Commercial $\gamma$-FeOOH (above 99\% purity) manufactured by Kojundo Chemical Lab. Co., Ltd., was used as pure $\gamma$-FeOOH particles in order to conduct a comparative study of the structure and morphology of the corrosion products obtained in practice. This is because the pure $\gamma$-FeOOH particles were relatively well crystallized, and they are hereafter referred to as $\gamma$-FeOOH.

\section{B. Measurements}

X-ray diffraction measurements were carried out using Mo $\mathrm{K} \alpha$ radiation with energy of $17.447 \mathrm{keV}(50 \mathrm{kV}, 30$ $\mathrm{mA}$ ). The X-ray scattering intensity was measured with a wide range of wave vectors in order to obtain an accurate interference function for each sample. The atomicscale structure of the $\gamma$-FeOOH particles was described using the partial distribution functions calculated from the interference functions obtained by statistical optimization through the RMC simulation technique [16-18]. The RMC routine is essentially identical to that used in our previous study $[9,12]$. The supercells of $\gamma$-FeOOH had dimensions of $3.756 \times 3.870 \times 3.684 \mathrm{~nm}^{3}$. The simulation was accomplished with initial configurations of 4320 atoms (1440 for iron and 2880 for oxygen) in each supercell of $\gamma$-FeOOH.

A TEM apparatus, JEOL JEM-1200EX II, was used for observing the microscopic morphology of the particles. The bonding structure of the particles was analyzed by Fourier-transform infrared spectroscopy (FT-IR; DIGILAB FTS 7000).

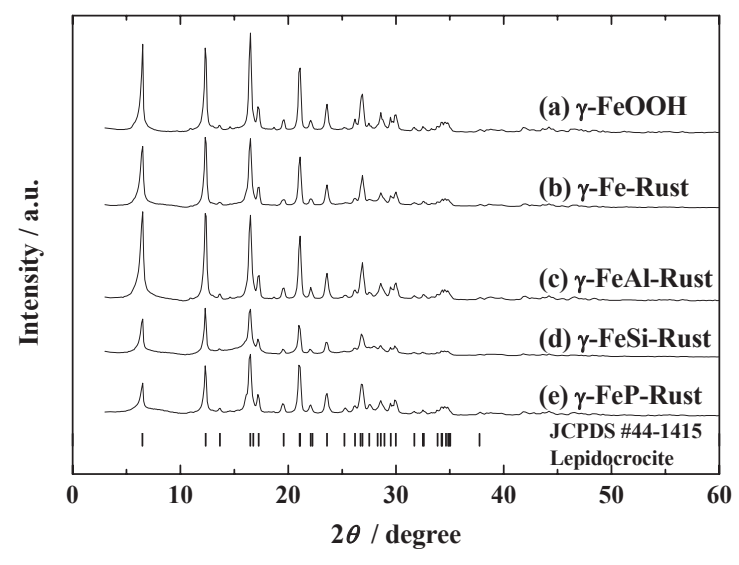

FIG. 1: X-ray diffraction patterns obtained with Mo K $\alpha$ radiation as a function of $2 \theta$ for (a) $\gamma$-FeOOH, (b) $\gamma$-Fe-Rust, (c) $\gamma$-FeAl-Rust, (d) $\gamma$-FeSi-Rust, and (e) $\gamma$-FeP-Rust. The reference lines given in the JCPDS database are also shown.

\section{RESULTS AND DISCUSSION}

\section{A. Atomic-scale structure characterization of different $\gamma$-FeOOH particles}

The X-ray diffraction patterns of $\gamma$-FeOOH, $\gamma$-Fe-Rust, $\gamma$-FeAl-Rust, $\gamma$-FeSi-Rust, and $\gamma$-FeP-Rust are shown in Figs. 1(a), (b), (c), (d) and (e), respectively. The reference diffraction pattern of $\gamma$-FeOOH provided by the JCPDS database is also shown. The results indicate that the diffraction peaks of these samples are fundamentally assigned to the $\gamma$-FeOOH structure, and the structures of $\gamma$-Fe-Rust, $\gamma$-FeAl-Rust, $\gamma$-FeSi-Rust, and $\gamma$-FeP-Rust seem to be relatively distorted as compared to the structure of $\gamma$-FeOOH.

Figures 2(a), (b), (c), (d) and (e) show the ordinary interference functions $Q_{i}(Q) / \mathrm{nm}^{-1}$ for $\gamma$-FeOOH, $\gamma$-FeRust, $\gamma$-FeAl-Rust, $\gamma$-FeSi-Rust, and $\gamma$-FeP-Rust, respectively. The experimental data are denoted by symbols, while the results calculated using the RMC simulation are plotted as solid lines. The RMC simulation was carried out for estimating the realistic atomic-scale arrangements in these samples. The interference functions computed by the RMC method were compared with the experimental interference functions so that new atomic configurations in the supercell were generated through the random movement of atomic positions for structural optimization. The interference functions were calculated by the repetition of acceptance and rejection on the basis of a comparison with the previous configuration data.

The partial pair distribution functions of the $\mathrm{Fe}-\mathrm{Fe}$ $\left(g_{\mathrm{Fe}-\mathrm{Fe}}\right), \mathrm{Fe}-\mathrm{O}\left(g_{\mathrm{Fe}-\mathrm{O}}\right)$, and $\mathrm{O}-\mathrm{O}\left(g_{\mathrm{O}-\mathrm{O}}\right)$ pairs in $\gamma-$ FeOOH, $\gamma$-Fe-Rust, $\gamma$-FeAl-Rust, $\gamma$-FeSi-Rust, and $\gamma$ FeP-Rust estimated by the RMC simulation are shown in Figs. 3(a)-(c), respectively. In the partial pair distribution functions of $g_{\mathrm{Fe}-\mathrm{Fe}}$, the first peak is located at approximately $0.3 \mathrm{~nm}$ in $\gamma-\mathrm{FeOOH}$, while the same peak in $\gamma$-Fe-Rust, $\gamma$-FeAl-Rust, $\gamma$-FeSi-Rust, and $\gamma$-FeP-Rust become broad distribution. The additional peaks appear at $0.27 \mathrm{~nm}$ and $0.35 \mathrm{~nm}$ for $\gamma$-Fe-Rust, $\gamma$-FeAl-Rust, $\gamma$-FeSiRust, and $\gamma$-FeP-Rust. Although the first peak is located at approximately $0.2 \mathrm{~nm}$ for $\gamma-\mathrm{FeOOH}$ in Fig. 3(b), the 


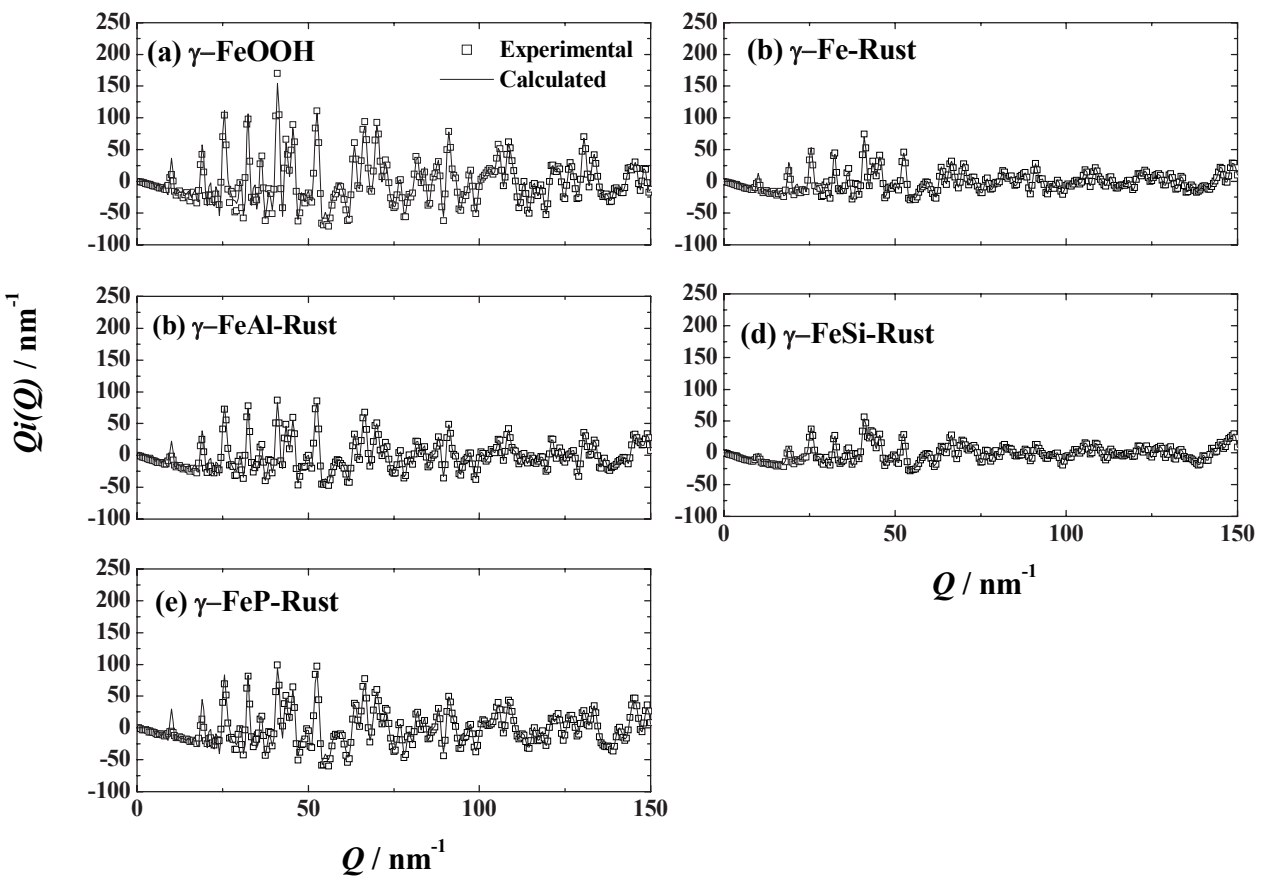

FIG. 2: The ordinary interference function $Q_{i}(Q)$ for (a) $\gamma$-FeOOH, (b) $\gamma$-Fe-Rust, (c) $\gamma$-FeAl-Rust, (d) $\gamma$-FeSi-Rust, and (e) $\gamma$-FeP-Rust. The dots and solid lines indicate the experimental data and values calculated by RMC simulation, respectively.

splitting of peaks at $0.2 \mathrm{~nm}$ are observed for $\gamma$-Fe-Rust, $\gamma$-FeAl-Rust, $\gamma$-FeSi-Rust, and $\gamma$-FeP-Rust. Such a tendency is also observed in the pair distribution functions of O-O pairs $\left(g_{\mathrm{O}-\mathrm{O}}\right)$ for $\gamma$-Fe-Rust, $\gamma$-FeAl, $\gamma$-FeSi-Rust, and $\gamma$-FeP-Rust, as shown in Fig. 3(c). These results indicate that the fundamental $\mathrm{FeO}_{6}$ octahedral units and their linkages appear to be distorted in $\gamma$-Fe-Rust, $\gamma$-FeAlRust, $\gamma$-FeSi-Rust, and $\gamma$-FeP-Rust. In particular, the appearance of the peak of $0.27 \mathrm{~nm}$ and $0.35 \mathrm{~nm}$ in Fig. 3(a) and the splitting of peaks at 0.2 for $\gamma$-Fe-Rust, $\gamma$-FeAlRust, $\gamma$-FeSi-Rust, and $\gamma$-FeP-Rust may be attributed to the formation of corner and face linkages of octahedral units like $\alpha-\mathrm{FeOOH}$ and $\beta$ - $\mathrm{FeOOH}$ [1]. These may arise from the incorporation of chlorine, aluminum, silicon, and phosphorus. However, the peaks in the partial pair distribution functions of $\gamma$-FeAl-Rust are relatively sharper than those for the other rusts. In $g_{\mathrm{Fe}-\mathrm{Fe}}$ of the partial pair distribution functions of $\gamma$-FeSi-Rust, a significant structural distortion is observed. Thus, it is considered that the atomic-scale distortion due to foreign ions depends on the ion species. For instance, silicon and phosphorus in alloys become silicate ions and phosphate ions in aqueous solutions; these ions may be adsorbed on to the surface of $\gamma$-FeOOH particles or incorporated into them. This adsorption or incorporation is likely to induce the distortion of the linkages of the fundamental $\mathrm{FeO} 6$ octahedral units in $\gamma$-FeSi-Rust and $\gamma$-FeP-Rust.

Figures 4(a)-(e) show the realistic atomic arrangements calculated by the RMC simulation for $\gamma$-FeOOH, $\gamma$-FeRust, $\gamma$-FeAl-Rust, $\gamma$-FeSi-Rust, and $\gamma$-FeP-Rust, respectively. The ideal $\gamma$-FeOOH structure consists of double chains of edge-shared octahedral units parallel to the baxis, as shown in Fig. 4(a). Such a structure is also observed in the corrosion products: $\gamma$-Fe-Rust, $\gamma$-FeAl-Rust, $\gamma$-FeSi-Rust, and $\gamma$-FeP-Rust. However, the structure of the $\mathrm{FeO}_{6}$ octahedral units and their linkages of these units

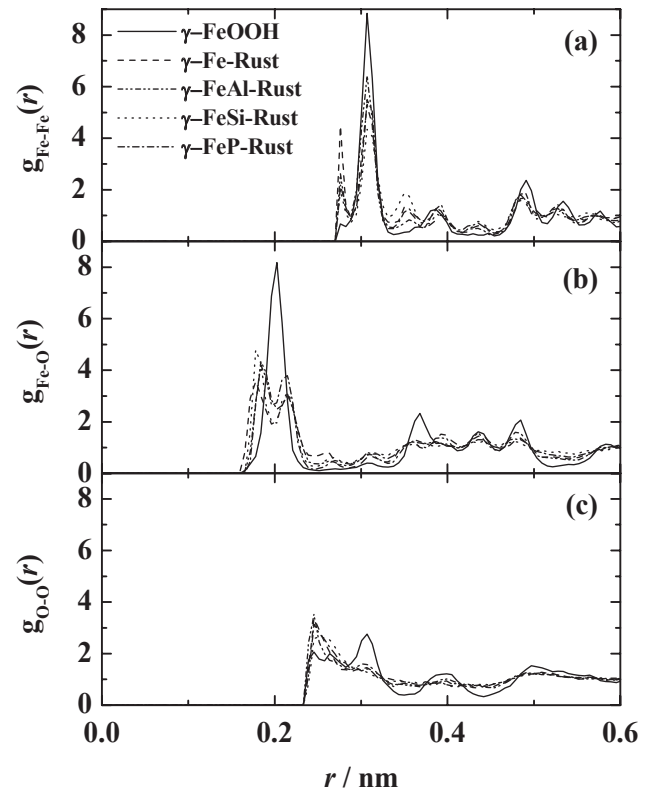

FIG. 3: Partial pair distribution functions of (a) Fe-Fe, (b) $\mathrm{Fe}-\mathrm{O}$, and (c) O-O pairs in $\gamma$-FeOOH, $\gamma$-Fe-Rust, $\gamma$-FeAl-Rust, $\gamma$-FeSi-Rust, and $\gamma$-FeP-Rust estimated by RMC simulation.

in the samples are dependent on the alloying elements. The atomic-scale structure appears to be distorted in the following order: $\gamma$-FeAl-Rust $<\gamma$-Fe-Rust $<\gamma$-FeP-Rust $<\gamma$-FeSi-Rust.

\section{B. Transmission electron micrographs and infrared spectroscopy}

As the microscopic morphology of the $\gamma-\mathrm{FeOOH}$ particles is reflected by their atomic-scale structure, the TEM 
(a) $\gamma$-FeOOH

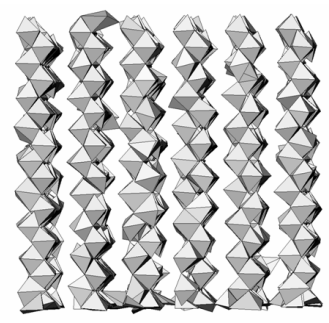

(c) $\gamma$-FeAl-Rust

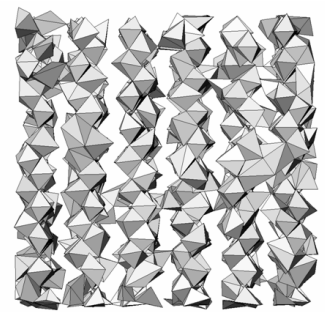

(e) $\gamma$-FeP-Rust

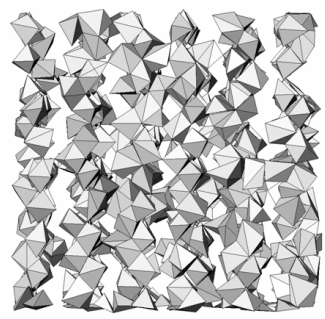

FIG. 4: Atomic arrangements of (a) $\gamma$-FeOOH, (b) $\gamma$-Fe-Rust, (c) $\gamma$-FeAl-Rust, (d) $\gamma$-FeSi-Rust, and (e) $\gamma$-FeP-Rust using the $\mathrm{FeO}_{6}$ octahedral structural units estimated by RMC simulation.

images of $\gamma$-FeOOH, $\gamma$-Fe-Rust, $\gamma$-FeAl, $\gamma$-FeSi-Rust and $\gamma$-FeP-Rust are taken, as shown in Fig. 5. The plate-like morphology of these samples is attributed to the layered structure of $\gamma-\mathrm{FeOOH}$, as shown in Fig. 4. The size and aspect ratio of the particles depend on the sample. The morphologies of $\gamma$-Fe-Rust and $\gamma$-FeAl-Rust are clearly plate-like, whereas those of $\gamma$-FeSi-Rust and $\gamma$-FeP-Rust deviate from the plate-like shape. Thus, the morphology of the $\gamma$-FeOOH particles is likely to be related with the atomic-scale distortion obtained from the quantitative X-ray diffraction analysis and RMC simulation. The differences in the atomic-scale structure and morphology are induced by the incorporation of foreign ions into the samples.

Figure 6 shows the FT-IR spectra of the samples measured in the $500-1500 \mathrm{~cm}^{-1}$ region. Four main absorption peaks are observed at approximately 1142, 1023, 750, and $583 \mathrm{~cm}^{-1}$, which is the characteristic of the bending structure in $\gamma$-FeOOH [19-21]. The absorption band at approximately $1142 \mathrm{~cm}^{-1}$ is assigned to the in-plane Fe$\mathrm{O}-\mathrm{H}$ bending vibration. It shifts to a higher wave number in $\gamma$-Fe-Rust, $\gamma$-FeAl-Rust, $\gamma$-FeSi-Rust, and $\gamma$-FePRust. In $\gamma$-FeSi-Rust, the peak at approximately 1142 $\mathrm{cm}^{-1}$ is the broadest. The absorption band at approximately $583 \mathrm{~cm}^{-1}$ is attributed to the $\mathrm{Fe}-\mathrm{O}$ vibration related to the displacement of oxygen ions $\left(\mathrm{O}^{2-}\right)$, and this band is smaller in $\gamma$-Fe-Rust, $\gamma$-FeAl-Rust, $\gamma$-FeSi-Rust,
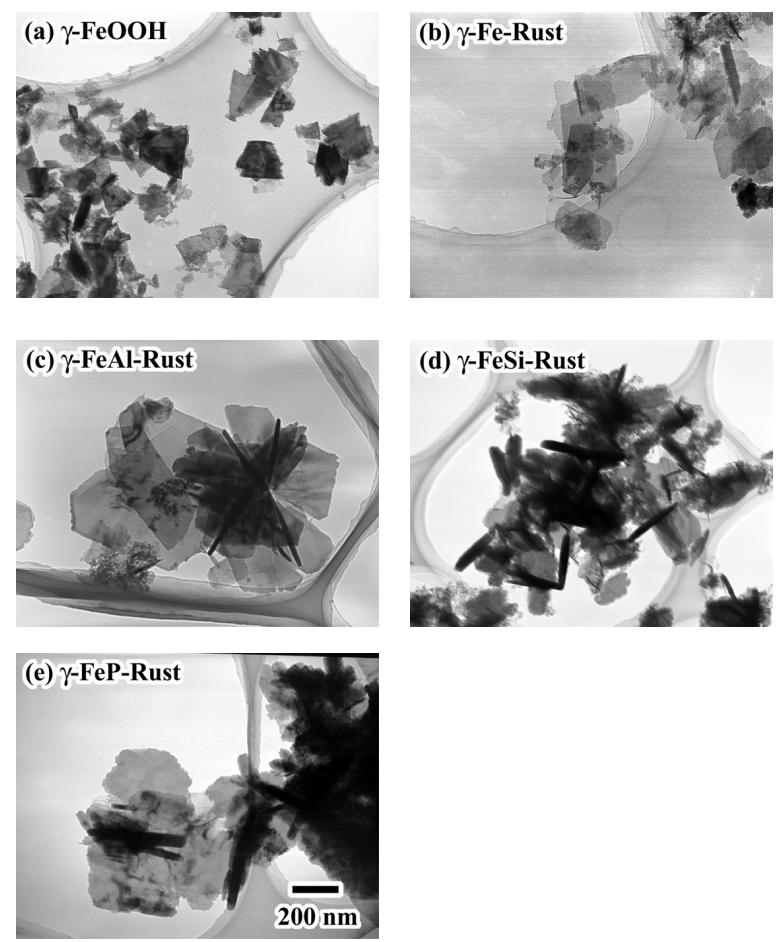

FIG. 5: TEM images of (a) $\gamma$-FeOOH, (b) $\gamma$-Fe-Rust, (c) $\gamma$ FeAl-Rust, (d) $\gamma$-FeSi-Rust, and (e) $\gamma$-FeP-Rust.

and $\gamma$-FeP-Rust. These results show that the bonding structures in in-plane $\mathrm{Fe}-\mathrm{O}-\mathrm{H}$ bending and $\mathrm{Fe}-\mathrm{O}$ vibration have changed in $\gamma$-Fe-Rust, $\gamma$-FeAl-Rust, $\gamma$-FeSi-Rust and $\gamma$-FeP-Rust. In addition, the adsorption band at approximately $910 \mathrm{~cm}^{-1}$ is observed in $\gamma$-FeSi-Rust, which is caused by the Fe-O-Si bending vibration $[19,20]$. In contrast, the absorption bands at approximately $1023 \mathrm{~cm}^{-1}$ and $750 \mathrm{~cm}^{-1}$ in $\gamma$-FeAl-Rust, which are assigned to the out-plane O-H bending [1], are sharper than those of other samples. This result may be related to the characteristic structure and morphology of $\gamma$-FeAl-Rust, although the influence of aluminum should be further studied.

In this study, the influence of foreign ions on X-ray scattering was neglected for structural estimation since the atomic positions of the cations and anions in the unit cell are not identified and they are of relatively light el-

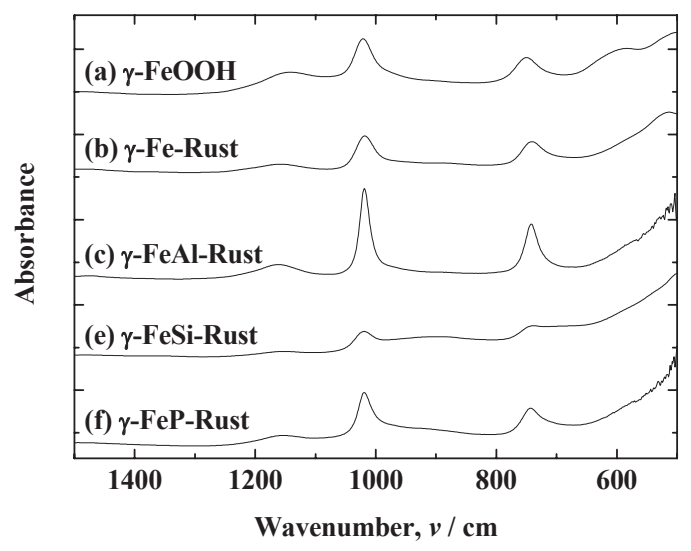

FIG. 6: FT-IR spectra of (a) $\gamma$-FeOOH, (b) $\gamma$-Fe-Rust, (c) $\gamma$-FeAl-Rust, (d) $\gamma$-FeSi-Rust, and (e) $\gamma$-FeP-Rust. 
ements. Nevertheless, the atomic-scale structures estimated by the RMC simulation in this study are believed to be sufficiently reasonable.

\section{CONCLUSION}

The influence of aluminum, silicon, and phosphorus on the structure of $\gamma$-FeOOH particles formed during the corrosion of iron alloys were characterized by quantitative Xray structural analysis along with RMC simulation. TEM observations and IR measurements were also performed.

1. The atomic arrangements and pair distribu- tion functions estimated using quantitative X-ray diffraction patterns along with the RMC simulation indicated that the linkages of $\mathrm{FeO}_{6}$ octahedral units in $\gamma$-FeOOH were distorted by the incorporation of foreign elements. This distortion increased in following order: $\gamma$-FeAl-Rust $<\gamma$-Fe-Rust $<\gamma$-FePRust $<\gamma$-FeSi-Rust.

2. FT-IR spectra suggest that the foreign elements incorporated from the alloys were present in the $\gamma$ FeOOH particles. This possibly led to a morphological change in the particles, as observed in the TEM images.
[1] M. Cornel and U. Schwertmann, The Iron Oxides (WileyVCH, Weinheim, 2003).

[2] J. P. Jolivet, C. Chaneac, and C. Fiaud, Chem. Commun. 5, 481 (2004).

[3] C. Leygraf and T. Graedel, Atmospheric Corrosion (Wiley-Interscience, New York, 2000).

[4] T. Misawa, M. Yamashita, K. Matsuda, H. Miyuki, and H. Nagano, Iron Steel Inst. Jpn. 79, 69 (1993).

[5] T. Okada, et al., Jpn. J. Appl. Phys. 39, 3382 (2000).

[6] K. Shinoda, E. Matsubara, A. Muramatsu, and Y. Waseda, Mater Trans. JIM. 35, 394 (1994).

[7] S. Suzuki, M. Saito, M. Kimura, T. Suzuki, H. Kihira, and Y. Waseda, ISIJ International 43, 366 (2003).

[8] S. Suzuki, Y. Takahashi, M. Saito, M. Kusakabe, T. Kamimura, H. Miyuki, and Y. Waseda, Corros. Sci. 47, 1271 (2005).

[9] S. K. Kwon, S. Suzuki, M. Saito, and Y. Waseda, Corros. Sci. 47, 2543 (2005)

[10] K. Kanie, A. Muramatsu, S. Suzuki, and Y. Waseda, Mater. Trans. 45, 968 (2004).

[11] S. K. Kwon, K. Kimijima, K. Kanie, A. Muramatsu, S. Suzuki, E. Matsubara, and Y. Waseda, ISIJ International
45, 77 (2005).

[12] K. Inoue, S. K. Kwon, S. Suzuki, M. Sitou, and Y. Waseda, Mater. Trans. 47, 243 (2006).

[13] S. Suzuki, T. Suzuki, M. Kimura, Y. Takagi, K. Shinoda, K. Tohji, and Y. Waseda, Appl. Surf. Sci. 169, 109 (2001).

[14] M. Yamashita, T. Shimizu, H. Konishi, J. Mizuki, and H. Uchida, Corros. Sci. 45, 381 (2003).

[15] S. Suzuki, Y. Takahashi, T. Kamimura, H. Miyuki, Y. Takagi, K. Shinoda, K. Tohji, and Y. Waseda, Corros. Sci. 46, 1751 (2004).

[16] Y. Waseda, Anomalous X-ray Scattering for Materials Characterization (Springer-Verlag, Heidelberg, 2002).

[17] M. Saito, S. Kang, and Y. Waseda, J. Phys. Sci. Jpn. 68, 1932 (1999).

[18] S. Kang, C. Park, M. Saito, and Y. Waseda, Mater. Trans. JIM 40, 552 (1999)

[19] D. G. Lewis and V. C. Farmer, Clays Miner. 21, 93 (1986).

[20] R. Raman, B. Kuban, and A. Razvan, Corros. Sci. 32, 1295 (1991).

[21] B. Weckler and H. D. Lutz, Eur. J. Solid State Inorg. Chem. 35, 531 (1998). 